\title{
Graphene-Based Sensors for Clinical Analysis
}

\author{
Livia Alexandra Gugoasa* \\ Laboratory of Electrochemistry, Romania
}

*Corresponding author: Livia Alexandra Gugoasa, Laboratory of Electrochemistry and PATLAB, National Institute of Research for Electrochemistry and Condensed Matter, 202 Splaiul Independent Str., 060021, Bucharest-6, Romania. Email: livia.gugoasa@yahoo.com

To Cite This Article: Livia Alexandra Gugoasa. Graphene-Based Sensors for Clinical Analysis . Am J Biomed Sci \& Res. 2019 - 3(4). AJBSR. MS.ID.000695. DOI: 10.34297/AJBSR.2019.03.000695

Received: June 17, 2019 | Published: June 25, 2019

\begin{abstract}
Graphene presents great interestas sensor material. Its exceptional physical and chemical properties made it a star material for the electrochemical detection of different analytes from biological samples. The applications in which graphene-based materials were involved generated thousands of papers but in this mini review we sum only very few of them.
\end{abstract}

Keywords: Graphene, Sensors, Biological samples, Clinical analysis, Electrochemical detection

\section{Introduction}

Graphene sheet, a monocrystalline graphitic film is much stable and of remarkably high quality than graphite and have attracted a significant attention from an experimental and theoretical view [1]. Graphene sheets are considered fascinating materials due to their high specific surface area, good conductivity and excellent mechanical strength [2]. Nowadays, graphene can be fabricated to use it in effective ways and make it easy for researches to use it in the design of electrochemical sensors [3] and as matrix in the design of composite materials ("graphene based"): graphene sheets mixed with Co hexacyanoferrate nanoparticles (GS-CoNP) [4], mixed with methylene blue (MB) and chitosan (CS) as organic component (GSMB-CS) for the assay of prostate specific antigen. GS-MB enhances the conductivity and offers a larger specific surface area which helps the antibody immobilization process [5].

CuO-graphene sheets nanocomposite has been built by physical deposition of $\mathrm{CuO}$ nanocubes onto graphene sheets. This method of fabrication is simpler and time saving. The nanocomposite presents a good ability to promote the electron transfer reactions and enhanced electrocatalytic activity, which makes it a good choice for glucose sensing [6].

Graphene`s derivates, graphene oxide (GO) and reduced graphene oxide (rGO) are also excellent materials used in electrochemical sensors' design, due to their larger surface area. The modification of structural functional groups makes possible to improve the selectivity aspect of the electrochemical sensors [7].

Graphene oxide and Au nanoclusters (AuNCs) composite was prepared using ? ? 0 国stacking method between GO and AuNCs. The nanocomposite exhibited excellent features such as water solubility, biocompatibility and good stability. Due to these properties and because it provided a good platform for the interaction between the analyte and electrode, GO-AuNCs was used for the assay of L-cysteine [8].

Another nanocomposite using graphene sheets was prepared by adding an electroactive component, thionine (Th). This GSTh nanocomposite increases antibody loading and makes the development of graphene-based immunosensors, easier, for the assay of ?fetoprotein (AFP) [9].

In the last years modified rGO composites were used as material in the design of stochastic sensors [10-13]. Platinum and gold graphene composite pastes were mixed with a 10-3 mol L-1 solution of protoporphyrin IX for the screening of saliva samples for simultaneous determination of leptin, plasminogen activator inhibitor 1 (PAI-1), interleukin 6 and monocyte chemotactic protein 1 (MCP-1) [10]. Ag-TiO2 graphene composite was used for molecular recognition of carcinoembryonic antigen in blood samples [11]. Pyridine and porphyrin modification of Ag-TiO2 and $\mathrm{Au}-\mathrm{TiO} 2$ graphene composite paste was investigated for

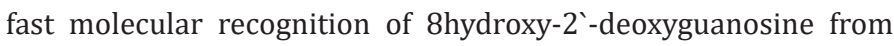
biological samples [12]. A three dimensional (3D) printed Au-rGO composite paste electrode was studied for the electrochemical determination of BPA from saliva samples. The Au-rGO composite powder was mixed with paraffin oil to form a homogenous paste, obtaining the working paste electrode [13].

\section{Conclusion}

Graphene GO and rGO are intensively studied materials in the electrochemical field due to its high conductivity, biocompatibility 
and stability. In the last years researchers used this material for lots of applications such as clinical analysis, with very good results, making graphene a star material in sensors` design.

\section{Acknowledgements}

This work was financially supported by the Ministry of Research and Innovation, CNCSUEFISCDI, under grant number PN-III-P1-1.1PD2016-0190, within PNCDI III.

\section{References}

1. Geim AK, Novoselov KS (2007) The rise of graphene. Nature Materials 6: 183-191.

2. Stankovich S, Dikin DA, Dommet GHB, Kohlhaas KM, Zimney EJ et al. (2006) Graphene-based composite materials. Nature 442(7100): 282 286.

3. Wu F, Xu MQ Zhao GC (2010) Graphene-based modified electrode for the direct electron transfer of Cytochrome $\mathrm{c}$ and biosensing. Electrochemistry Communications 12(1): 175-177.

4. Li T, Yang M, Li H (2011) Label-free electrochemical detection of cancer marker based on graphene-cobalt hexacyanoferrate nanocomposite. Journal of Electroanalytical Chemistry 655(1): 50-55.

5. Mao K, Wu D, Li Y, Ma H, Ni Z, et al. (2012) Label-free electrochemical immunosensor based on graphene/methylene blue nanocomposite. Anal Biochem 422(1): 22-27.

6. Luo L, Zhu L, Wang Z (2012) Nonenzymatic amperometric determination of glucose by $\mathrm{CuO}$ nanocubes-graphene nanocomposite modified electrode. Bioelectrochemistry 88: 156-163.
7. Shanbhag VKL, Prasad KS (2016) Graphene based sensors in the detection of glucose in saliva - a promising emerging modality to dignose diabetes mellitus. Analytical Methods 8(33): 6255-6259.

8. Ge S, Yan M, Lu J, Zhang M, Yu F, et al. (2012) Electrochemical biosensor based on graphene oxide-Au nanoclusters composites for L-cysteine analysis. Biosens Bioelectron 31(1): 49-54.

9. Wei Q Mao K, Wu D, Dai Y, Yang J, et al. (2010) A novel label-free electrochemical immunosensor based on graphene and thionine nanocomposite. Sens Actuators B Chem 149(1): 314-318.

10. Gugoasa LA, Stefan van Staden RI, Dima A, Visan CA, Streinu Cercel A, Biris A, et al. (2015) Fast screening of biological fluids for cytokines and adipokines using stochastic sensing. Microelectronic Engineering 148: 64-69.

11. Gugoasa LA, Muklive Al Ogaidi AJ, Stefan van Staden RI, El Khatib A, Rosu MC, et al. (2017) Multimode microsensors based on Ag-TiO2- graphene materials used for the molecular recognition of carcinoembryonic antigen in whole blood samples. RSC Advances 7(45): 28419-28426.

12. Stefan van Staden RI, Balahura LR, Gugoasa LA, Aboul Enein H, van Staden JF, et al. (2018) Pattern recognition of 8-hydroxy-2 deoxyguanosine in biological fluids. Anal Bioanal Chem 410: 115-121.

13. Gugoasa LA, Stefan van Staden RI, van Staden JF, Coros M, Pruneanu M (2019) Electrochemical determination of bisphenol A in saliva by a novel three-dimensional (3D) printed gold-reduced graphene oxide (rGO) composite paste electrode. Analytical Letters. 